Cita: Pires, D. A.; Ferreira, R. W.; Vasconcelos, A. S. B.; Penna, E. M. (2019). Dimensões de Burnout, Estratégias de Coping e Tempo de Prática como Atleta Federado em Jogadores Profissionais de Futebol. Cuadernos de Psicología del Deporte, Vol 19(2), 175-185

\title{
Dimensões de Burnout, Estratégias de Coping e Tempo de Prática como Atleta Federado em Jogadores Profissionais de Futebol
}

\section{Dimensiones de Agotamiento, Estrategias de Afrontamiento y Tiempo de Práctica como Atleta Federado en Jugadores Profesionales de Fútbol}

\section{Burnout Dimensions, Coping Strategies and Practice Time as a Federated Athlete in Professional Soccer Players}

\author{
Pires, D. A. ${ }^{1}$, Ferreira, R. W. ${ }^{1}$, Vasconcelos, A. S. B. ${ }^{1}$, Penna, E. M. ${ }^{1}$ \\ ${ }^{1}$ Faculdade de Educação Física, Campus Universitário de Castanhal, Universidade Federal do Pará
}

\begin{abstract}
RESUMO
O objetivo do estudo consiste em analisar se há correlação entre as seguintes variáveis em jogadores profissionais de futebol: a) dimensões de burnout $\mathrm{x}$ estratégia de coping; b) tempo de prática como atleta federado $\mathrm{x}$ dimensões de burnout; c) tempo de prática como atleta federado $\mathrm{x}$ estratégias de coping. Participaram do estudo 54 atletas profissionais de futebol da primeira divisão do Campeonato Paraense de 2018, todos do sexo masculino. A média de idade foi de $24.0 \pm 3.9$ anos, e a média do tempo de prática como atleta federado foi de $8.7 \pm 4.2$ anos. Os participantes responderam a dois instrumentos psicométricos de mensuração de burnout e coping. Os resultados evidenciam que: i) os indicadores de burnout foram baixos; ii) a correlação entre as dimensões de burnout e coping foi significativa, negativa e moderada somente entre a dimensão reduzido senso de realização esportiva e a estratégia treinabilidade; iii) não observamos correlação entre o tempo de prática como atleta federado e as dimensões de burnout; iv) encontramos uma correlação significativa, positiva e moderada entre o tempo de prática como atleta federado e a estratégia desempenho sob pressão. As principais conclusões do estudo apontam que atletas com maior tempo de prática como federados tendem a apresentar melhor desempenho sob pressão, bem como o desenvolvimento da estratégia treinabilidade pode ser oportuno para a percepção de realização esportiva em atletas.
\end{abstract}

Palavras-chave: Psicologia do esporte, Habilidades psicológicas, Treinabilidade.

\section{RESUMEN}

El objetivo del estudio consiste en analizar si hay correlación entre las siguientes variables en jugadores profesionales de fútbol: a) dimensiones de agotamiento $\mathrm{x}$ estrategia de afrontamiento; $\mathrm{b}$ ) tiempo de práctica como atleta federado $\mathrm{x}$ dimensiones de agotamiento; c) tiempo de práctica como atleta federado $\mathrm{x}$ estrategias de afrontamiento. 


\title{
Pires, D. A.; Ferreira, R. W.; Vasconcelos, A. S. B.; Penna, E. M.
}

Participaron del estudio 54 atletas profesionales Brasileños de fútbol de la primera división del Campeonato Paraense de 2018, todos del sexo masculino. El promedio de edad fue de $24.0 \pm 3.9$ años, y el promedio del tiempo de práctica como atleta federado fue de $8.7 \pm 4.2$ años. Los participantes respondieron a dos instrumentos psicométricos de medición de agotamiento y afrontamiento. Los resultados evidencian que: i) los indicadores de agotamiento fueron bajos; ii) la correlación entre las dimensiones de agotamiento y afrontamiento fue significativa, negativa y moderada solamente entre la dimensión reducido sentido de realización deportiva y la estrategia de capacitación; iii) no observamos correlación entre el tiempo de práctica como atleta federado y las dimensiones de agotamiento; iv) encontramos una correlación significativa, positiva y moderada entre el tiempo de práctica como atleta federado y la estrategia de rendimiento bajo presión. Las principales conclusiones del estudio apuntan que los atletas con mayor tiempo de práctica como federados tienden a presentar mejor desempeño bajo presión, así como el desarrollo de la estrategia de capacitación puede ser oportuno para el sentido de realización deportiva en atletas.

Palabras clave: Psicología del deporte, Habilidades psicológicas, Capacitación.

\begin{abstract}
The objective of the study is to analyze whether there is correlation between the following variables in professional soccer players: a) burnout dimensions x coping strategies; b) practice time as a federated athlete $\mathrm{x}$ burnout dimensions; c) practice time as a federated athlete $\mathrm{x}$ coping strategies. Fifty-four professional Brazilian soccer athletes from the first division of the 2018 Pará State Championship, all males, participated in the study. The mean age was $24.0 \pm 3.9$ years, and the mean time of practice as a federated athlete was $8.7 \pm 4.2$ years. Participants responded to two paper and pencil psychometric instruments measuring burnout and coping. The results show that: i) the burnout indicators were low; ii) the correlation between the dimensions of burnout and coping was significant, negative and moderate only between the dimension reduced sense of accomplishment and the strategy of coachability; iii) we did not observe correlation between the time of practice as a federated athlete and the dimensions of burnout; iv) we found a significant, positive and moderate correlation between the practice time as a federated athlete and the strategy performance under pressure. The main conclusions of the study point out that athletes with higher practice time as federated tend to present better performance under pressure, as well as the development of the strategy coachability may be opportune for athletes' sense of accomplishment.
\end{abstract}

Keywords: Sport psychology, Psychological skills, Coachability.

\section{INTRODUÇÃO}

Os atletas estão expostos a várias circunstâncias geradoras de estresse e a maneira com que cada um enfrenta o mesmo poderá interferir no seu desempenho (Christensen \& Smith, 2018; Rossi, Vitorino, Salles, \& Cortez, 2016). Sendo assim, ao se tornar crônico, o estresse no contexto esportivo pode ocasionar a síndrome de burnout (Gustafsson, DeFreese, \& Madigan, 2017), que tem como algumas consequências ausência de motivação, falta de prazer, altos níveis de estresse e enfrentamento ineficaz diante os agentes estressores (Goodger, Gorely, Lavallee, \& Harwood, 2007).

O burnout no contexto esportivo é definido como uma síndrome multidimensional baseada em três dimensões (Gustafsson, Lundkvist, Podlog, \& Lunqvist, 2016): exaustão física e emocional (EFE), relacionada com intensas demandas de treinos e competições; reduzido senso de realização esportiva (RSR), que significa a insatisfação relacionada à habilidade e realização no âmbito esportivo; e desvalorização esportiva (DES), representando a ausência de interesse e preocupação com a carreira esportiva. Os estudos sobre a síndrome de burnout têm aumentado nos últimos anos, pois sua ocorrência pode resultar em prejuízos para o rendimento esportivo e para a saúde dos atletas, sendo o abandono da carreira esportiva a principal consequência (García-Parra, González, \& de los Fayos, 2016).

Por se tratar de uma reação ao estresse crônico, a manifestação do burnout pode ser prevenida ou atenuada por meio de estratégias de enfretamento do 


\section{Burnout e Coping em Jogadores Profissionais de Futebol}

estresse, conhecidas como coping (Pires, Bara Filho, Debien, Coimbra, \& Ugrinowitsch, 2016). O coping é um recurso utilizado mediante pensamento e ação como forma de lidar com situações estressantes (Lazarus, 1993). Uma das primeiras nomenclaturas para a classificação das estratégias de coping foi proposta por Folkman \& Lazarus (1980). Os autores propuseram duas modalidades de coping: (1) centrada no problema, que envolve o enfrentamento do problema causador de estresse; e (2) centrada na emoção, que objetiva aliviar as emoções negativas associadas ao problema.

As estratégias centradas no problema são empregadas pelos atletas com o intuito de resolver o problema, assumir o controle e eliminar a situação estressante. A meta-análise realizada por Nicholls, Taylor, Carroll, \& Perry (2016) apontou que as estratégias de coping centradas no problema obtiveram uma relação positiva com o rendimento esportivo. Outro tipo de estratégia é a centrada na emoção, que são tentativas de avaliar de outra maneira ou evitar pensar no agente estressor (Lazarus, 1993). Estas estratégias incluem esquiva, escape, distanciamento e extração de aspectos positivos de acontecimentos negativos. São utilizadas quando o sujeito percebe que o estressor não pode ser modificado e que é preciso continuar com a interação (Tamayo \& Tróccoli, 2002). Diferentemente das estratégias centradas no problema, Nicholls et al. (2016) observaram que as estratégias centradas na emoção não são adequadas para o rendimento esportivo, bem como podem ser prejudiciais para o bem-estar dos atletas.

Estudo de revisão sistemática (Nicholls \& Polman, 2007) apontou que instrumentos psicométricos de identificação e mensuração das estratégias de coping são empregados com frequência na literatura. Sendo um dos mais frequentes o Inventário de Habilidades de Enfrentamento Atlético-28 (ACSI-28), desenvolvido para avaliar sete estratégias de coping utilizadas por atletas para lidar com o estresse no esporte (Smith, Schutz, Smoll, \& Ptacek, 1995), posteriormente validado para o idioma português como ACSI-28BR (Miranda, Coimbra, Bara Filho, Miranda Júnior, \& Andrade, 2018).

As sete habilidades de enfretamento medidas pelo ACSI-28 foram definidas por Smith \& Christensen (1995): a) Lidar com adversidades: o atleta permanece positivo e entusiasmado diante de situações adversas.
Permanecendo calmo, controlado e capaz de se recuperar rapidamente de erros e contratempos; b) Desempenho sob pressão: o esportista percebe a pressão como um desafio em vez de ameaça. O esportista gosta de ser colocado sob pressão e geralmente tem um desempenho melhor nessas ocasiões; c) Metas/Preparação Mental: o atleta define e trabalha para metas específicas de desempenho. Planeja e se prepara mentalmente para diversas situações que pode se deparar; d) Concentração: o sujeito não fica distraído facilmente e é capaz de se concentrar na tarefa mesmo em situações adversas ou inesperadas; e) Livre de preocupação: o atleta não se pressiona e não coloca preocupação desnecessária sobre o seu desempenho, mesmo quando comete erros. Além disso, não se importa sobre o que vão pensar se ele falhar; f) Confiança e motivação: essa habilidade é definida quando o esportista está confiante sobre suas capacidades, permanece sempre motivado e positivo sobre seu desempenho; g) Treinabilidade: capacidade do atleta estar disposto a aprender com as instruções dos treinadores e receber as críticas de uma maneira construtiva, sem ficar chateado e levar para o lado pessoal.

A síndrome de burnout está associada à percepção de que as estratégias de coping são insuficientes ou inadequadas para lidar com o estresse (Verardi, Nagamine, Domingos, De Marco, \& Miyazaki, 2015). Raedeke \& Smith (2004) verificaram que atletas que experimentam altos níveis de estresse e possuem baixos índices nas estratégias de coping estão mais propensos a experimentar o burnout. Diante disso, é importante que os esportistas desenvolvam estratégias de coping para que possam enfrentar os diversos agentes estressores e assim possibilitar condições ideais para um bom desempenho esportivo e êxito na carreira esportiva (Nascimento Junior, Gaion, Nakashima, \& Vieira, 2010). Para tais circunstâncias Wong, Teo, \& Polman (2015) ressaltam a importância da compreensão por parte dos treinadores e psicólogos do esporte sobre os agentes estressores e das estratégias de coping como forma de otimizar o desempenho dos atletas nos treinamentos $\mathrm{e}$ competições.

O futebol é um esporte que apresenta um alto nível de estresse devido à procura de ótimo rendimento, pois consiste em uma modalidade esportiva com visibilidade e exibição diária nos meios de 


\section{Pires, D. A.; Ferreira, R. W.; Vasconcelos, A. S. B.; Penna, E. M.}

comunicação nacional e internacional (Giacomoni \& Fonseca, 2014). Nesse contexto, a torcida, dirigentes e até mesmo os próprios jogadores fazem cobranças por conquistas de vitórias e títulos, levando a um aumento nos níveis de estresse (Bemfica, Fagundes, Pires, \& Costa, 2013). Vale ressaltar que ainda são escassos os estudos que investigam os aspectos psicológicos no contexto do futebol na região norte do Brasil (Oliveira, Penna, \& Pires, 2017). Além disso, estudo recente de revisão sistemática sobre o burnout aponta para a relevância da investigação sobre a variável tempo de prática esportiva e sua possível influência na percepção de burnout em atletas de elite (Bicalho \& Costa, 2018). Sendo assim, o presente estudo tem como objetivo analisar se há correlação entre as seguintes variáveis em jogadores profissionais de futebol: a) dimensões de burnout $\mathrm{x}$ estratégia de coping; b) tempo de prática como atleta federado $\mathrm{x}$ dimensões de burnout; c) tempo de prática como atleta federado $\mathrm{x}$ estratégias de coping.

\section{MATERIAIS E MÉTODOS}

\section{Participantes}

Participaram do estudo 54 jogadores profissionais de futebol do sexo masculino, atuantes na primeira divisão do Campeonato Paraense de 2018 (a média de idade foi de $24.0 \pm 3.9$ anos, a idade média em que iniciaram na modalidade foi de $11.5 \pm 3.8$ anos e a média do tempo de prática como atleta federado foi de $8.7 \pm 4.2$ anos). Foram selecionados para participar do estudo somente atletas que possuíam no mínimo um ano de prática como atleta federado (a contar do primeiro registro).

\section{Instrumentos}

Foi utilizada a versão em idioma português do Athlete Burnout Questionnaire (ABQ) (Raedeke \& Smith, 2001), denominada Questionário de Burnout para Atletas (QBA), composta por 15 itens cujas respostas são apresentadas em uma escala do tipo likert que varia de "quase nunca" (1) a "quase sempre" (5). O QBA apresenta consistência interna aceitável (alfa de Cronbach $=0.82$ ) e validade de construto, na qual foram encontrados três componentes responsáveis pela variância do instrumento, confirmando o modelo tridimensional do burnout (Pires, Brandão, \& Silva,
2006). Logo, tem sido utilizado com frequência no contexto esportivo brasileiro (Pires et al., 2016; Silva, Chiminazzo, \& Pires, 2016; Sobral, Oliveira, Oliveira, Santos, \& Brito, 2014).

Outro instrumento utilizado foi o Athletic Coping Skills Inventory (ACSI-28) (Smith et al., 1995) em sua versão adaptada para o Português (ACSI-28BR) (Miranda et al., 2018). O ACSI-28BR é composto por 28 itens que se referem aos sete fatores que representam estratégias de coping no esporte: lidar com adversidades; desempenho sob pressão; metas/preparação mental; concentração; livre de preocupação; confiança/motivação; e treinabilidade. As respostas são apresentadas em uma escala do tipo likert que varia de "quase nunca" $(0)$ a "quase sempre" (3). Especificamente para a versão brasileira, três itens associados às dimensões confiança/motivação, concentração e treinabilidade foram removidos por não apresentarem cargas fatoriais suficientes.

\section{Procedimentos}

Inicialmente foi realizado um contato prévio com a comissão técnica dos clubes para esclarecimento dos procedimentos do estudo. Após esse contato houve a coleta dos dados em grupo com cada time em salas reservadas de hotéis e centros de treinamento em ambientes livres de ruído. Os pesquisadores explicaram os objetivos da pesquisa aos participantes, que posteriormente assinaram o Termo de Consentimento Livre e Esclarecido (TCLE), preencheram o questionário sociodemográfico e o QBA e ACSI-28BR. O presente estudo foi aprovado pelo Comitê de Ética em Pesquisa (CEP) com seres humanos, conforme parecer 65796316.3.0000.0017.

\section{Análise estatistica}

A verificação da normalidade da distribuição dos dados foi realizada por meio do teste de D'AgostinoPearson. $O$ teste apontou uma distribuição de não normalidade dos dados, o que determinou o emprego de testes não-paramétricos. Como os dados não apresentaram distribuição normal, foram utilizados mediana $(\mathrm{Md})$ e quartis $(\mathrm{Q} 1$; $\mathrm{Q} 3)$ para a caracterização dos resultados. O coeficiente de correlação de Spearman (rho) foi empregado para verificar as correlações entre os indicadores das dimensões de burnout e das estratégias de coping, bem como as correlações entre o tempo de prática como atleta 


\section{Burnout e Coping em Jogadores Profissionais de Futebol}

federado e os indicadores de burnout e coping. Foram considerados a significância estatística e um valor mínimo crítico de rho $\geq 0.40$ como aceitável para as análises, pois corresponde ao limite inferior da intensidade moderada para a correlação entre variáveis (Dancey \& Reidy, 2013; Thomas, Nelson, \& Silverman, 2012). Os procedimentos estatísticos foram realizados no pacote estatístico Prisma, versão 7 (GraphPad Software Inc., San Diego, CA, EUA). O nível de significância adotado foi de $\mathrm{p}<0.05$.

\section{RESULTADOS}

A Tabela 1 apresenta os dados descritivos das dimensões de burnout, estratégias de coping e tempo de prática como atleta federado. Os resultados evidenciam que as dimensões de burnout ficaram com uma frequência de quase nunca a raramente, mostrando que os atletas estão pouco suscetíveis à síndrome. A estratégia de coping com maior mediana foi metas/preparação mental e a menor foi lidar com adversidades.

Tabela 1. Dados descritivos para as dimensões de burnout, estratégias de coping e tempo de prática como atleta federado (em anos).

\begin{tabular}{|c|c|c|c|c|c|c|c|c|c|c|c|}
\hline & \multicolumn{3}{|c|}{ Burnout } & \multicolumn{7}{|c|}{ Coping } & \multirow[b]{2}{*}{ TPF } \\
\hline & EFE & RSR & DES & LA & DP & $\mathbf{P M}$ & $\mathrm{CO}$ & $\mathbf{L P}$ & CM & TR & \\
\hline Mín & 1.0 & 1.0 & 1.0 & 4.0 & 4.0 & 4.0 & 3.0 & 0.0 & 4.0 & 0.0 & 1.0 \\
\hline Q1 & 1.4 & 1.6 & 1.4 & 7.0 & 6.7 & 8.0 & 5.0 & 4.0 & 7.0 & 5.0 & 5.0 \\
\hline Md & 1.8 & 1.8 & 1.8 & 9.0 & 9.0 & 9.5 & 6.0 & 5.0 & 8.0 & 6.0 & 8.0 \\
\hline Q3 & 2.2 & 2.2 & 2.0 & 10.0 & 11.0 & 11.0 & 7.0 & 7.0 & 9.0 & 8.0 & 12.0 \\
\hline Máx & 5.0 & 3.4 & 5.0 & 12.0 & 12.0 & 12.0 & 9.0 & 10.0 & 9.0 & 9.0 & 20.0 \\
\hline
\end{tabular}

$\mathrm{Na}$ Tabela 2 foram encontradas correlações negativas, significativas e de intensidade fraca (rho $<0.40$ ): dimensão EFE x estratégia confiança e motivação; dimensão RSR x estratégia metas/ preparação mental; dimensão DES x estratégia treinabilidade. A única correlação negativa, significativa e de intensidade moderada $(0.40 \leq$ rho $<0.70)$ foi observada entre a dimensão RSR e a estratégia treinabilidade. 


\section{Pires, D. A.; Ferreira, R. W.; Vasconcelos, A. S. B.; Penna, E. M.}

A Tabela 3 apresenta os resultados das correlações entre o tempo de prática como atleta federado e os indicadores de burnout e estratégias de coping. Foi observada uma correlação positiva, significativa e de intensidade fraca (tempo de prática como atleta

Tabela 2. Correlação entre as dimensões de burnout e estratégias de coping.

\begin{tabular}{cccccccc}
\hline & LA & DP & PM & CO & LP & CM & TR \\
\hline EFE & -0.07 & -0.02 & -0.15 & -0.15 & 0.20 & $-0.34^{*}$ & -0.15 \\
RSR & -0.01 & -0.12 & $-0.30^{*}$ & -0.01 & 0.22 & -0.25 & $-0.40^{*}$ \\
DES & -0.09 & 0.02 & -0.02 & -0.20 & -0.12 & -0.02 & $-0.28^{*}$
\end{tabular}

$\mathrm{EFE}=$ Exaustão física e emocional; RSR= Reduzido senso de realização esportiva; $\mathrm{DES}=$ Desvalorização esportiva; $\mathrm{LA}=$ Lidar com as adversidades; $\mathrm{DP}=$ Desempenho sob pressão; $\mathrm{PM}=$ Preparação mental; $\mathrm{CO}=$ Concentração; $\mathrm{LP}=$ livre de preocupação; $\mathrm{CM}=$ Confiança/Motivação; $\mathrm{TR}=$ Treinabilidade; $\mathrm{TPF}=\mathrm{Tempo}$ de prática como atleta federado. $* \mathrm{p}<0,05$.

Tabela 3. Correlação do tempo de prática como atleta federado (em anos) entre as dimensões de burnout e estratégias de coping.

federado x estratégia lidar com adversidades) e uma correlação positiva, significativa e de intensidade moderada (tempo de prática como atleta federado $\mathrm{x}$ dimensão desempenho sob pressão).

\begin{tabular}{|c|c|c|c|c|c|c|c|c|c|c|}
\hline & \multicolumn{3}{|c|}{ Burnout } & \multicolumn{7}{|c|}{ Coping } \\
\hline & EFE & RSR & DES & LA & DP & $\mathbf{P M}$ & $\mathrm{CO}$ & LP & CM & TR \\
\hline TPF & 0.03 & 0.02 & -0.06 & $0.38^{*}$ & $0.54 *$ & 0.18 & 0.18 & 0.13 & -0.02 & -0.07 \\
\hline
\end{tabular}

$\mathrm{TPF}=$ Tempo de prática como atleta federado; $\mathrm{EFE}=$ Exaustão física e emocional; RSR= Reduzido senso de realização esportiva; $\mathrm{DES}=$ Desvalorização esportiva; $\mathrm{LA}=$ Lidar com as adversidades; $\mathrm{DP}=$ Desempenho sob pressão; $\mathrm{PM}=$ Preparação mental; $\mathrm{CO}=$ Concentração; $\mathrm{LP}=$ livre de preocupação; $\mathrm{CM}=$ Confiança/Motivação; $\mathrm{TR}=$ Treinabilidade. ${ }^{*} \mathrm{p}<0,05$.

\section{DISCUSSÃO}

O presente estudo analisou as seguintes correlações em jogadores profissionais de futebol: a) dimensões de burnout $\mathrm{x}$ estratégia de coping; b) tempo de prática como atleta federado $\mathrm{x}$ dimensões de burnout; c) tempo de prática como atleta federado $\mathrm{x}$ estratégias coping. Os resultados evidenciam que: i) os indicadores de burnout foram baixos; ii) a correlação entre as dimensões de burnout e coping foi significativa, negativa e moderada somente entre a dimensão RSR e a estratégia treinabilidade; iii) não observamos correlação entre o tempo de prática como atleta federado e as dimensões de burnout; iv) encontramos uma correlação significativa, positiva e moderada entre o tempo de prática como atleta federado e a estratégia desempenho sob pressão.

Os achados de que atletas de futebol apresentam baixos indicadores para as dimensões de burnout podem ser indicativos de uma adaptação adequada às demandas psicofisiológicas de treinos e competições, bem como de um equilíbrio entre o estresse e a recuperação, relacionada à dimensão EFE (Giacomoni \& Fonseca, 2014). Os baixos escores para a dimensão RSR sugerem que os jogadores estão satisfeitos com suas habilidades e realizações no âmbito esportivo. Por fim, a percepção reduzida de DES pode indicar 


\section{Burnout e Coping em Jogadores Profissionais de Futebol}

que os jogadores permanecem interessados e comprometidos com a carreira esportiva (Bemfica et al., 2013; Gustafsson, DeFreese, \& Madigan, 2017). Os dados descritivos das dimensões de burnout estão em consonância com a literatura da psicologia do esporte, pois a maior parte da pesquisa esportiva é baseada em amostras de atletas endossando níveis baixos a moderados de sintomas de burnout, a partir dos escores do ABQ (Gustafsson, DeFreese, \& Madigan, 2017).

A maior parte das correlações entre as dimensões de burnout e as estratégias de coping foi de magnitude fraca, apontando para a compreensão dessas variáveis como fenômenos distintos. Uma possível explicação para esses achados está no fato de que os mesmos não indicam que essas estratégias não sejam utilizadas de forma eficaz pelos atletas em algum momento do ciclo de treinamento, uma vez que as estratégias de enfrentamento podem mudar ao longo da temporada. O mesmo raciocínio pode ser aplicado à percepção das dimensões de burnout (Pires et al., 2016).

A única exceção foi verificada na correlação entre a dimensão RSR e a estratégia treinabilidade, que apresentou significância estatística, relação negativa e magnitude moderada. Com base neste resultado é possível sugerir que estar aberto e disponível para a aprendizagem a partir dos treinamentos, exercitar a habilidade em lidar com as cobranças e evitar considerar as críticas da comissão técnica como ofensas pessoais contribuem para a percepção de satisfação relacionada à habilidade e ao desempenho no futebol (Pires, 2014). Estudo envolvendo atletas de elite do futsal brasileiro apontou que atletas convocados para a seleção brasileira utilizam com mais frequência a estratégia de treinabilidade do que seus pares que não foram convocados (Bim, Nascimento Junior, Amorim, Vieira, \& Vieira, 2014), reforçando a relação dessa estratégia com a percepção de sucesso esportivo.

Não observamos correlação entre o tempo de prática como atleta federado e as dimensões de burnout. Vieira, Carruzo, Aizava, \& Rigoni (2013) também não encontraram correlação entre tais variáveis em atletas de vôlei de praia. De acordo com os autores, as diferenças nas percepções das dimensões de burnout podem estar mais vinculadas a diferenças no perfil psicológico das pessoas, como características de personalidade (Hill, Hall, Appleton, \& Murray, 2010) e de motivação (Lonsdale, Hodge, \& Rose, 2009), do que simplesmente ao tempo de prática como atleta federado.

Por outro lado, os resultados encontrados em outra investigação mostram que, quanto maior o tempo de prática, menores são os níveis dos sintomas relacionados à síndrome de burnout em jovens atletas de vôlei feminino (Teixeira, Batista, Leite, \& Silva, 2016). De modo divergente aos estudos anteriores, Cresswell \& Eklund (2006) e Toros (2018) apontaram que, quanto maior a experiência, mais percebidas são as dimensões EFE e DES em atletas de rúgbi e handebol, respectivamente.

Diante dos resultados heterogêneos, entendemos que a variável tempo de prática como atleta federado não possui relação relevante com a percepção da síndrome de burnout em atletas profissionais de futebol. Essa compreensão encontra respaldo no estudo de revisão sistemática de Goodger et al. (2007), na qual o tempo de experiência não foi listado como variável associada ao burnout em atletas, diferentemente de outras variáveis como motivação, coping e ansiedade.

Diferentemente dos resultados observados em relação às dimensões de burnout, o tempo de prática como atleta federado apresentou correlação significativa, positiva e moderada com a estratégia de coping desempenho sob pressão. Vieira et al. (2013) também verificaram que, quanto mais experiente é o atleta, menos ele será prejudicado com a pressão de uma competição. Tais descobertas sugerem que as habilidades psicológicas de coping podem ser desenvolvidas com o tempo de prática (Mahl \& Raposo, 2007). Para Kent, Devonport, Lane, Nicholls, \& Friesen (2018), a simples exposição repetida a situações de pressão tendem a melhorar o rendimento do atleta. Desse modo, o tempo de prática pode estar associado à diversidade de tarefas sob pressão vivenciadas no contexto esportivo no decorrer da carreira, o que pode ocasionar maior oportunidade de desenvolver habilidades de coping centradas no problema (em termos gerais) e a estratégia desempenho sob pressão (de modo específico).

Este estudo utilizou instrumentos psicométricos recorrentemente utilizados na literatura para dimensionar burnout e coping. Contudo, entrevistas qualitativas poderiam fornecer informações subjetivas sobre as experiências individuais de burnout e coping, 


\section{Pires, D. A.; Ferreira, R. W.; Vasconcelos, A. S. B.; Penna, E. M.}

bem como de suas interações. Sua utilização em estudos futuros pode oferecer informações complementares sobre os fenômenos investigados.

\section{APLICAÇÕES PRÁTICAS}

O processo de treinamento associado ao esporte competitivo acaba por colocar os atletas sob agentes estressores físicos e psicológicos (Madigan, Hill, Anstiss, Mallinson-Howard, \& Kumar, 2018). Diante disso, os atletas estão suscetíveis ao burnout quando há um desequilíbrio entre as demandas pertinentes à sua participação no esporte e os recursos que têm para enfrentar tais demandas (Pacewicz, Gotwals, \& Blanton, 2018).

Os resultados deste estudo podem dar indícios para a atuação dos profissionais que trabalham com esporte. $\mathrm{Na}$ perspectiva prática, o desenvolvimento de intervenções de treinamento das estratégias de coping mostrou efeito positivo em atletas ao incrementar a percepção de desempenho (Reeves, Nicholls, \& Mckenna, 2011) e pode representar uma iniciativa gratificante para aqueles interessados em promover o bem-estar do atleta através da prevenção e tratamento do burnout. Os resultados do presente estudo sugerem que a estratégia de enfrentamento treinabilidade pode ser aperfeiçoada com o intuito de evitar o burnout em geral e a dimensão RSR em particular. Além disso, os resultados também deixaram indicativos para a investigação da percepção da síndrome em atletas de diferentes níveis de experiência e de como as estratégias de coping variam ao longo da temporada, levando em consideração as especificidades da organização das competições e das modalidades esportivas.

\section{REFERÊNCIAS}

1. Bemfica, T., Fagundes, L., Pires, D., \& Costa, V. (2013). Efeito da temporada sobre a percepção da síndrome de burnout por atletas de futebol profissional. Revista Brasileira de Ciência e Movimento, 21(4), 142-150. DOI: 10.18511/0103-1716/rbcm.v21n4p142-150

2. Bicalho, C., \& Costa, V. (2018). Burnout in Elite Athlete/s: a Systematic Review.
Cuadernos de Psicología del Deporte, 18(1), 89-102.

3. Bim, R., Nascimento Junior, J., Amorim, A., Vieira, J., \& Vieira, L. (2014). Estratégias de coping e sintomas de burnout em atletas de futsal de alto rendimento. Revista Brasileira de Ciência e Movimento, 22(3), 69-75. DOI: 10.18511/0103-1716/rbcm.v22n3p69-75

4. Christensen, D., \& Smith, R. (2018). Leveling the playing field: can psychological coping resources reduce the influence of physical and technical skills on athletic performance? Anxiety, Stress, \& Coping, 1-13. DOI:10.1080/10615806.2018.1506646

5. Cresswell, S., \& Eklund, R. (2006). Changes in athlete burnout over a thirty-week "rugby year". Journal of Science and Medicine in Sport, $\quad 9$ 125-134. DOI:10.1016/j.jsams.2006.03.017

6. Dancey, C., \& Reidy, J. (2013). Estatística sem matemática para a psicologia. 5a ed. Porto Alegre: Penso.

7. Freudenberger, H. Staff Burnout. (1974). Journal of Social Issues, 30(1), 159-166.

8. Folkman, S., \& Lazarus, R. (1980). An Analysis of Coping in a Middle-Aged Community Sample. Journal of Health and Social Behaviour, 21(3), 219-239.

9. García-Parra, N., González, J., \& de los Fayos, E. (2016). Estado actual del estudio del síndrome de burnout en el deporte. Cuadernos de Psicología del Deporte, 16(2), 21-28.

10. Giacomoni, C., \& Fonseca, G. (2014). Principais Indicadores de Burnout em Jovens Atletas de Futebol. Revista Mackenzie de Educação Física e Esporte, 13(2), 106-123.

11. Goodger, K., Gorely, T., Lavallee, D., \& Harwood, C. (2007). Burnout in Sport: A Systematic Review. The Sport Psychologist, 21, 127-151. DOI: 10.1123/tsp.21.2.127 


\section{Burnout e Coping em Jogadores Profissionais de Futebol}

12. Gustafsson, H., Defreese, J., \& Madigan, D. (2017). Athlete burnout: review and recommendations. Current Opinion in Psychology, 16, 109-113. DOI: 10.1016/j.copsyc.2017.05.002

13. Gustafsson, H., Lundkvist, E., Podlog, L., \& Lundqvist, C. (2016). Conceptual Confusion and Potential Advances in Athlete Burnout Research. Perceptual and Motor Skills, 123(3), 784-791. DOI: $10.1177 / 0031512516665900$

14. Hill, A., Hall, H., Appleton, P., \& Murray, J. (2010). Perfectionism and burnout in canoe polo and kayak slalom athletes: the mediating influence of validation and growth-seeking. The Sport Psychologist, 24,16-34. DOI: $10.1123 /$ tsp.24.1.16

15. Kent, S., Devonport, T., Lane, A., Nicholls, W., \& Friesen, A. (2018). The Effects of Coping Interventions on Ability to Perform Under Pressure. Journal of Sports Science and Medicine, 17, 40-55.

16. Lazarus, R. (1993). From Psychological Stress to the Emotions: A History of Changing Outlooks. Annual Review of Psychology, 44, $1-21$.

17. Lonsdale, C., Hodge, K., \& Rose, E. (2009). Athlete burnout in elite sport: a selfdetermination perspective. Journal of Sports Sciences, 27, 785-95. DOI: 10.1080/02640410902929366

18. Mahl, A., \& Raposo, J. (2007). Perfil psicológico de prestação de jogadores

profissionais de futebol do Brasil. Revista Portuguesa de Ciências do Desporto, 7(1), 8091.

19. Madigan, D., Hill, A., Anstiss, P., MallinsonHoward, S., \& Kumar, S. (2018). Perfectionism and training distress in junior athletes: The mediating role of coping tendencies. European Journal of Sport
Science, $18(5), \quad 713-721 . \quad$ DOI: 10.1080/17461391.2018.1457082

20. Miranda, R., Coimbra, D., Bara Filho, M., Miranda Júnior, M., \& Andrade, A. (2018). Brazilian Version (ACSI-28BR) of Athletic Coping Skills Inventory-28. Revista Brasileira de Medicina do Esporte, 24(2), 130-134. DOI: $10.1590 / 1517-869220182402160980$

21. Nascimento Junior, J., Gaion, P., Nakashima, F., \& Vieira, L. (2010). Análise do estresse psicológico pré-competitivo estratégias de coping de jovens atletas de futebol de campo. Revista Brasileira de Ciência e Movimento, 18(4), 45-53.

22. Nicholls, A. \& Polman, R. (2007). Coping in sport: A systematic review. Journal of Sports Sciences, 25(1), 11-31. DOI: $10.1080 / 02640410600630654$

23. Nicholls, A., Taylor, N., Carroll, S., \& Perry, J. (2016). The Development of a New SportSpecific Classification of Coping and a MetaAnalysis of the Relationship between Different Coping Strategies and Moderators on Sporting Outcomes. Frontiers in Psychology, 7, 1-14. DOI: 10.3389/fpsyg.2016.01674

24. Oliveira, A., Penna, E., \& Pires, D. (2017). Síndrome de Burnout em Árbitros de Futebol. Revista de Psicología del Deporte, 27(1), 3136.

25. Pacewicz, C., Gotwals, J., \& Blanton, J. (2018). Perfectionism, coping, and burnout among intercollegiate varsity athletes: A person-oriented investigation of group differences and mediation. Psychology of Sport \& Exercise 35, 207-217. DOI: 10.1016/j.psychsport.2017.12.008

26. Pires, D. (2014). Estudo longitudinal dos indicadores da síndrome de burnout e das estratégias de coping em atletas brasileiros de alto rendimento. Tese de doutorado, Universidade Federal de Minas Gerais, Belo Horizonte, MG, Brasil. 


\section{Pires, D. A.; Ferreira, R. W.; Vasconcelos, A. S. B.; Penna, E. M.}

27. Pires, D., Bara Filho, M., Debien, P., Coimbra, D., \& Ugrinowitsch, H. (2016). Burnout e Coping em Atletas de Voleibol: uma análise longitudinal. Revista Brasileira de Medicina do Esporte, 22(4), 277-281. DOI: $10.1590 / 1517-869220162204158756$

28. Pires, D., Brandão, M., \& Silva, C. (2006). Validação do questionário de burnout para atletas. Revista da Educação Física/UEM, 17(1), 27-36.

29. Raedeke, T., \& Smith, A. (2004). Coping Resources and Athlete Burnout: An Examination of Stress Mediated and Moderation Hypotheses. Journal of Sport \& Exercise Psychology, 26, 525-541. DOI: $101123 /$ jsep.26.4.525

30. Raedeke, T., \& Smith, A. (2001). Development and Preliminary Validation of an Athlete Burnout Measure. Journal of Sport and Exercise Psychology, 23(4), 281-306. DOI: $101123 /$ jsep.23.4.281

31. Reeves, C., Nicholls, A., \& Mckenna, J. (2011). The effects of a coping intervention on coping self-efficacy, coping effectiveness, and subjective performance among adolescent soccer players. International Journal of Sport and Exercise Psychology, 9(2), 126-42. DOI: 10.1080/1612197X.2011.567104

32. Rossi, M., Vitorino, L., Salles, R., \& Cortez, P. (2016). Estratégias de Coping em Atletas de Futebol Feminino: Estudo Comparativo. Revista Brasileira de Medicina do Esporte, 22(4), 282-286. DOI: $10.1590 / 1517-$ 869220162204160575

33. Silva, E., Chiminazzo, J., \& Pires, D. (2016). Indicadores da síndrome de burnout em atletas amadores brasileiros de rugby sevens. Pensar a Prática, 19(1), 92-102. DOI: 10.5216/rpp.v19i1.37019

34. Smith, R., \& Christensen, D. (1995). Psychological Skills as Predictors of Performance and Survival in Professional
Baseball. Journal of Sport \& Exercise Psychology, 17(4), 399-415.

35. Smith, R., Schutz, R., Smoll, F., \& Ptacek, J. (1995). Development and Validation of a multidimensional measure of sport-specific psychological skills: the athletic coping skills inventory-28. Journal of Sport \& Exercise Psychology, 17(4), 379-98. DOI: 101123/jsep.17.4.374

36. Sobral, A., Oliveira, L., Oliveira, L. S., Santos, R., \& Brito, A. (2014). Associação entre as cargas de treino impostas a jogadores amadores de rugby sevens e a síndrome de burnout. Motricidade, 10(2), 25-35. DOI: 10.6063/motricidade.10(2).2592

37. Tamayo, M., \& Tróccoli, B. (2002). Exaustão emocional: relações com a percepção de suporte organizacional e com as estratégias de coping no trabalho. Estudos de Psicologia, $7(1), \quad 37-46 . \quad$ DOI: $10.1590 / \mathrm{S} 1413-$ 294X2002000100005

38. Teixeira, M., Batista, M., Leite, L., Silva, S. (2016). Síndrome de burnout em atletas infanto-juvenís e juvenís de voleibol feminino participantes do campeonato brasileiro de seleções. Journal of Physical Education, 27(1). DOI: 10.4025/jphyseduc.v27i1.2717

39. Thomas, J., Nelson, J., \& Silverman, S. (2012). Métodos de Pesquisa em Atividade Física. $6^{\mathrm{a}}$ ed. Porto Alegre: Artmed.

40. Toros, T. (2018). Burnout Levels of Handball Players with Respect to Age, Gender and Experience. Asian Journal of Education and Training, 4(1), 29-34. DOI: 10.20448/journal.522.2018.41.29.34

41. Verardi, C., Nagamine, K., Domingos, N., De Marco, A., \& Miyazaki, M. (2015). Burnout and pre-competition: A study of its occurrence in Brazilian soccer players. Revista de Psicología del Deporte, 24(2), 259-264.

42. Vieira, L., Carruzo, N., Aizava, P., \& Rigoni, P. (2013). Análise da síndrome de "burnout" e das estratégias de "coping" em atletas 


\section{Burnout e Coping em Jogadores Profissionais de Futebol}

brasileiros de vôlei de praia. Revista Brasileira de Educação Física e Esporte, 27(2), 269-276. DOI: $10.1590 / \mathrm{S} 1807-55092013005000012$

43. Wong, R., Teo, E., \& Polman, R. (2015). Stress, coping, coping effectiveness and emotions in Malaysian Elite tenpin bowlers: Role of context and importance. International Journal of Sport and Exercise Psychology, 13(4), 320-334. DOI: 10.1080/1612197X.2014.999345 